\title{
CHARLES EHRESMANN'S CONCEPTS IN DIFFERENTIAL GEOMETRY
}

\author{
PAULETTE LIBERMANN \\ Université Denis Diderot (Paris VII) \\ 116 avenue du Général Leclerc, 75014 Paris, France
}

To the memory of Charles Ehresmann

\begin{abstract}
We outline some of the tools C. Ehresmann introduced in Differential Geometry (fiber bundles, connections, jets, groupoids, pseudogroups). We emphasize two aspects of C. Ehresmann's works: use of Cartan notations for the theory of connections and semi-holonomic jets.
\end{abstract}

Introduction. As R. Hermann puts it "Ehresmann's work was very prophetic and provided a general framework for describing Geometry and the mathematics which have a geometric component".

One of the motivations of C. Ehresmann to build up the foundations of Differential Geometry was to understand Elie Cartan's work from a global point of view. He was also influenced by S. Lie and E. Vessiot.

Besides his written work, C. Ehresmann had a great influence in the development of Geometry. His lectures, his seminars (where well known mathematicians of many countries, as well as young mathematicians like Thom, Reeb, Kuiper, Wu Wen Tsun, ... explained their results), his private conversations (where generously he gave new ideas) had played a significant part in this development.

The written part of Ehresmann's work in Differential Geometry and Algebraic Topology is published in the first volume (parts 1 and 2) of his "Euvres complètes" [6]. This volume also contains a report of C. Ehresmann on his own work (written in 1955) and comments from W. Van Est (homogeneous spaces and Lie groups), M. Zisman (fiber bundles), G. Reeb (foliations), R. Thom (jets), P. Libermann (connections and Lie pseudogroups), J. Pradines (groupoids), R. Hermann (applications to Physics and control

2000 Mathematics Subject Classification: Primary 53C05; Secondary 58A20, 58H05.

Key words and phrases: fiber bundles, connections, pseudogroups, groupoids, jets (holonomic and semi-holonomic).

The paper is in final form and no version of it will be published elsewhere. 
theory), A. Haefliger (the whole work in Geometry); A. Haefliger has also written a paper on the birth of foliations, published in the book "Géométrie au XX-ème siècle" [24]. I also gave contributions in the same book and in the publication "Fibrés, fibrations et connexions".

That is why this paper will only contain a brief report on the main notions of Differential Geometry introduced by C. Ehresmann (section 1). In sections 2 and 3 we shall emphasize two aspects of C. Ehresmann's work. In section 2 (which has many links with the contribution of C. Marle to this volume [37]) we deal with the theory of connections as introduced by C. Ehresmann. His paper on "Infinitesimal connections" (Bruxelles 1950, [17]) is fundamental for the subsequent development of the modern theory of connections. We show how C. Ehresmann utilizing E. Cartan's notations can build in a few pages this theory, thanks to his geometrical insight. He introduces for the first time the notion of differentiable groupoid. But C. Ehresmann was not satisfied with these notations and one year later he introduced the jets.

Section 3 deals with semi-holonomic jets. While the notion of holonomic jet is now classical, the notion of non holonomic ans semi-holonomic jet seems mysterious to many mathematicians. We show with the help of examples that the theory of semi-holonomic jets appears naturally in Differential Geometry.

In this paper we shall suppose that manifolds and mappings are $C^{\infty}$.

1. Survey of C. Ehresmann's work in Differential Geometry. The main fields investigated by E. Cartan that influenced C. Ehresmann in Differential Geometry were

a) Lie groups, homogeneous spaces, theory of the moving frame, Riemannian geometry,

b) generalized spaces and connections,

c) the so-called "infinite groups" and the equivalence problem.

1.1. Trying to understand E. Cartan's connections from the global point of view, C. Ehresmann worked on fiber bundles (particular cases of such bundles appear in his thesis "the topology of certain homogeneous spaces" [7]). He was one of the founders of the theory of fiber bundles, independently of Whitney and Steenrod. The first Notes were published from 1941 to 1943 [11, 12, 13, 14]; one of them was written in collaboration with J. Feldbau. C. Ehresmann introduced the notions of locally trivial principal bundle and their associated bundles. Later the introduction of pseudogroups of transformations simplified the definitions. For the topological results see the papers of M. Zisman and A. Haefliger in the "CEuvres complètes" [6].

An important problem was the restriction of the structure group of a $\mathcal{G}$-principal bundle to a subgroup $G$. In particular if $H$ is the frame bundle of a manifold $M$ of dimension $n$, a reduction of the structure group $G L(n, \mathbb{R})$ was called "regular infinitesimal structure" by C. Ehresmann. Later these structures were called $G$-structures by S. Chern but the original idea comes from Ehresmann.

The author proved that the existence of a reduction of $\mathcal{G}$ is equivalent to the existence of a section in an associated bundle with standard fiber $\mathcal{G} / G$.

On a paracompact manifold there always exists a Riemannian structure, which is not the case for a metric of type $(p, q)$. For instance on a compact manifold of dimension 4 , 
there exists a metric of type $(3,1)$ if and only if the Euler-Poincaré characteristic is null. So $S^{4}$ and $P_{4}(\mathbb{R})$ cannot be models for the Relativity.

Studying the existence of a complex structure on a manifold $M$, of dimension $2 n$, C. Ehresmann has introduced the notion of almost complex structure corresponding to a reduction of $G L(2 n, \mathbb{R})$ to $G L(n, \mathbb{C})$. He has shown that the existence of an almost complex structure is equivalent to the existence of an almost symplectic structure (defined by a non-degenerate 2 -form). For instance the sphere $S_{6}$ admits an almost complex structure defined by means of Cayley numbers; this structure does not come from a complex structure and is not symplectic (the 2-form is not closed) [29]. The existence of a complex structure on $S_{6}$ is still an open problem. Notice that the notion of almost complex structure plays an important part in Gromov's theory of pseudo-holomorphic curves.

1.2. An "infinite group" in the sense of E. Cartan is a family $\Gamma$ of differentiable mappings defined on open subsets of $\mathbb{R}^{n}$, endowed with a partial composition law, each $f \in \Gamma$ being the solution of a differential system of order $q \geq 1$.

The global version introduced by C. Ehresmann was the notion of Lie pseudogroup, which needs the introduction of the notions of pseudogroup of transformations, of jet, and of groupoid.

The notion of pseudogroup of transformations underlies a Note published in 1943 [14] where for the first time a structure of differentiable manifold was defined by means of local charts. The notion was introduced in 1947 [16]. It is fundamental in the papers of C. Ehresmann. Such a pseudogroup is a family of local homeomorphisms (on a topoloical space) or local diffeomorphisms on a differential manifold satisfying axioms which generalize those of Veblen-Whitehead. This notion permits to define local structures as for example differentiable manifolds, fiber bundles, foliations. A local structure is defined by an atlas of local maps from a manifold endowed with a pseudogroup $\Lambda$ onto another manifold, the changes of charts belonging to the pseudogroup.

1.3. C. Ehresmann has introduced the notion of infinitesimal jet in order to obtain an intrinsic differential calculus on manifolds. For instance it permits to define differential systems as submanifolds of jet spaces. As A. Haefliger writes "The notion of jet is now so familiar that we forgot how much its introduction has permitted to set the fundamental problems in local and global Differential Geometry". For instance R. Thom utilized jets when investigating the problem of the singularities of differential mappings.

For the theory of prolongations see [19, 20, 21, 22].

1.4. The groupoids have been introduced in Differential Geometry by C. Ehresmann in his paper on connections [17] first when considering the groupoid of closed paths, then associated with a principal bundle (gauge groupoid as we shall see later). All authors do not agree on the definition of a Lie groupoid; for some authors it is a groupoid with differentiable composition laws. For C. Ehresmann a Lie groupoid must be locally trivial.

Many authors worked on groupoids (J. Pradines, P. Libermann, K. Mackenzie, R. Brown). The subject has enjoyed renewed vigor with the introduction of symplectic groupoids independently by A. Weinstein an M. Karasev [23]. 
The publications of A. Weinstein, C. Albert and P. Dazord and their coworkers have settled the foundations of the theory $[1,5]$. Many books and papers have been published on the subject but sometimes the initiator C. Ehresmann has been forgotten. For more details see [36].

1.5. On a manifold $M$, the set $J^{q}(\Gamma)$ of $q$-jets of all local diffeomorphisms belonging to a pseudogroup $\Gamma$ constitute a groupoid, subgroupoid of the groupoid $\Pi^{q}(M)$ of the $q$-jets of all local diffeomorphisms of $M$.

A Lie pseudogroup of order $q$ on a manifold $M$ is a pseudogroup of local diffeomorphisms such that there exists an integer $q$ satisfying the conditions

a) $J^{q}(\Gamma)$ is a submanifold of $\Pi^{q}(M)$,

b) $\Gamma$ is the set of all solutions of $J^{q}(\Gamma)$ considered as a differential system. See [19] IV.

There are two types of Lie pseudogroups.

a) The Lie pseudogroups of finite type satisfying the condition: there exists an integer $r \geq q$ such that $J^{r}(\Gamma)$ is locally isomorphic to $J^{r-1}(\Gamma)$. For instance the pseudogroup of all local isometries of a Riemannian manifold.

b) the other ones called pseudogroups of infinite type. For instance the pseudogroup of all local symplectomorphisms of a symplectic manifold.

In 1958, C. Ehresmann [22] proved two theorems concerning the pseudogroups of finite type.

a) The group of all global transformations belonging to $\Gamma$ is a Lie group (theorem already known for the isometries of a Riemannian manifold).

b) If moreover the manifold is compact and simply connected, there exists a Lie group $G$ acting on $M$ such that any $f \in \Gamma$ is the restriction of an element of $G$.

Since 1960, the theory of Lie pseudogroups has known a great development. D. Spencer [25] introduced cohomological methods which were utilized by Kuranishi, H. Godschmidt, V. Guillemin, S. Sternberg, A. Kumpera, B. Malgrange. Lately P. Molino and C. Albert published a book on the subject [2].

1.6. We shall not tackle the theory of foliations created by C. Ehresmann and G. Reeb. One important contribution of C. Ehresmann was the introduction of the holonomy groupoid.

\section{On Cartan-Ehresmann notations and connections}

2.1. From his first Notes $[8,10]$ where he studied E. Cartan's "generalized spaces", his lecture on Lie groups (at the Julia seminar) in 1937 [9], until his famous paper on connections ("Colloque de Bruxelles"1950) [17], C. Ehresmann used E. Cartan's notations.

These notations which are linked with physics look intuitive and permit simple formulae but, as we shall see, they are sometimes ambiguous.

This is why C. Ehresmann tried more satisfactory notations and introduced the theory of jets. First order jets permit to define in an intrinsic way tangent vectors to a manifold. This notion already underlied a Note [14] where for the first time a manifold was defined by means of an "atlas of local charts". 
C. Ehresmann explains Cartan's notations in his paper on connections.

A tangent vector to a differentiable manifold $M$ with origin $x$ is denoted by

$$
x+d x .
$$

The prolongation of $f: M \rightarrow N$ to the tangent vectors is still denoted by $f, i . e$.

$$
f(x+d x)=f(x)+d(f x) .
$$

Let $(x+d x, y+d y)$ be a tangent vector to the manifold $M_{1} \times M_{2}$; then for the prolongation of $g: M_{1} \times M_{2} \rightarrow N$ to tangent vectors, we have

$$
g(x+d x, y+d y)=g(x+d x, y)+g(x, y+d y) .
$$

In particular if $g(x, y)$ is written $x . y$, we get

$$
(x+d x) \cdot(y+d y)=x \cdot(y+d y)+(x+d x) \cdot y .
$$

These formulae may be interpreted by means of 1-jets as follows: for any imbedding $\gamma: I \subset \mathbb{R}$ from an interval $I$ containing 0 to a manifold $M$ the symbol $x+d x$ (with $x=\gamma(t))$ represents the 1 -jet $j_{0}^{1} \tilde{\gamma}$, with source 0 , target $x$, the function $\tilde{\gamma}$ being the map $\gamma \circ \tau_{t}$, where $\tau_{t}$ is the translation $t \rightarrow 0$. When $M$ is an affine space, we recover the affine map tangent to $\gamma$ at the point $x=\gamma(t)$.

We could use the symbol $x+d x$ for a mapping from an open set of $\mathbb{R}^{p}$ to $M$ if the mapping is an imbedding. In particular if $p=n=\operatorname{dim} M$, we get a frame with origin $x$.

2.2. In his paper on connections [17] (colloque de Bruxelles, 1950), C. Ehresmann has introduced the notion of differentiable groupoid associated to a principal bundle. This groupoid is now called "gauge groupoid".

Let $(P, \pi, M)$ be a principal $G$-bundle. C. Ehresmann uses the same notation $z$ for an element of $P$ and the diffeomorphism $C_{z}$ of $G$ onto the fiber $P_{x}=\pi^{-1}(x)$ (with $x=\pi(z)$ ) defined by $s \mapsto z s$. With this convention, for any pair $\left(z, z^{\prime}\right) \in P \times P$, the diffeomorphism $C_{z^{\prime}} C_{z}^{-1}$ from $P_{x}$ to $P_{x^{\prime}}$ is denoted by $z^{\prime} z^{-1}$. The set of all isomorphisms from a fiber of $P$ to another one is denoted by $P P^{-1}$. As $\left(z^{\prime} s\right)(z s)^{-1}=z^{\prime} z^{-1}$, the manifold $P P^{-1}$ is the quotient of $P \times P$ by the equivalence relation $\left(z, z^{\prime}\right) \sim\left(z s, z^{\prime} s\right)$. The composition of maps induces a groupoid structure on $P P^{-1}$. The set of units is the set of identity maps on each fiber; it can be identified with $M$ (i.e. each $x$ is identified with the identity map of $\left.P_{x}\right)$. We have $\alpha\left(z^{\prime} z^{-1}\right)=\pi(z), \beta\left(z^{\prime} z^{-1}\right)=\pi\left(z^{\prime}\right)$.

The mapping $P \times P \rightarrow P P^{-1}$ can be prolongated to tangent vectors $\left(z, z^{\prime}+d z^{\prime}\right)$. The image of $\left(z, z^{\prime}+d z^{\prime}\right)$ is denoted by $\left(z^{\prime}+d z^{\prime}\right) z^{-1}$. In particular the vector $(z+d z) z^{-1}$ (called infinitesimal displacement) is a vector tangent to the $\alpha$-fiber $P_{x}$ with origin the identity map of $P_{x}$.

The consideration of the set $\mathcal{A}\left(P P^{-1}\right)$ of all infinitesimal displacements has led $\mathrm{J}$. Pradines to introduce the notion of Lie algebroid, notion which underlies C. Ehresmann's work. Recall that a vector bundle $p: \mathcal{A} \rightarrow M$ is endowed with a Lie algebroid structure if it satisfies the following properties:

a) the vector bundle is equipped with a Lie algebra structure [, ] on its space of sections,

b) there exists a bundle map $\rho: \mathcal{A} \rightarrow T M$ (called the anchor map) which induces a Lie algebra homomorphism (also denoted $\rho$ ) from sections of $\mathcal{A}$ to vector fields on $M$, 
c) for any smooth function $f$ on $M$ and any pair $(\xi, \eta)$ of sections of $\mathcal{A}$, the following identity is satisfied

$$
[f \xi, \eta]=f[\xi, \eta]-(\rho(\eta)) f \xi .
$$

If $\rho$ is surjective, the Lie algebroid is said to be transitive.

The importance of this notion comes from the fact that the set $\mathcal{A}(\Phi)$ of all tangent vectors to the $\alpha$-fibers of a Lie groupoid $\Phi$, with origin a unit constitute a Lie algebroid. Conversely a Lie algebroid does not come necessarily from a Lie groupoid. It is the problem of integration of a Lie algebroid. Many mathematicians had worked on this problem. See for instance the book by K. C. H. Mackenzie [36].

Let us go back to the principal $G$-bundle $(P, \pi, M)$. As

$$
(z+d z) s(z s)^{-1}=(z+d z) z^{-1}
$$

any infinitesimal displacement may be considered as an element of $T P / G$, set of all tangent vectors to $P$ modulo the right translations. We deduce an isomorphism from $\mathcal{A}\left(P P^{-1}\right)$ onto $T P / G$. The isomorphism was called by J. Pradines the Ehresmann isomorphism. Later the vector bundle $T P / G \rightarrow M$ was called the Atiyah-Molino bundle.

Let $(E, \pi, M)$ be a locally trivial fiber bundle. C. Ehresmann has defined an infinitesimal connection on $(E, \pi, M)$ as a distribution of $n$-contact elements (where $n=\operatorname{dim} M$ ) which is transverse to the fibers $\pi^{-1}(x)$ and satisfies the path lifting property: for any smooth path $\gamma$ on $M$ joining $x_{0}=\gamma\left(t_{0}\right)$ to $x_{1}=\gamma\left(t_{1}\right)$, there exists a horizontal smooth path $\tilde{\gamma}$ on $E$ joining $y_{0} \in \pi^{-1}\left(x_{0}\right)$ to $y_{1} \in \pi^{-1}\left(x_{1}\right)$ which projects on $\gamma$ by $\pi$, the point $y_{0}$ being arbitrary in $\pi^{-1}\left(x_{0}\right)$.

C. Ehresmann did not introduce the words "vertical" for the bundle $V E=\operatorname{ker} T \pi$ (which is tangent to the fibers) and "horizontal" for the transverse distribution. We use this terminology which is convenient.

In the case of a principal $G$-bundle $(P, \pi, M)$, C. Ehresmann adds the condition: the horizontal distribution must be invariant under the right action of $G$.

C. Ehresmann shows that the connection associates an infinitesimal displacement to any vector tangent to $M$; so the principal connection is a lifting $\mathcal{C}: T M \rightarrow T P / G$; as the base $M$ is supposed to be paracompact the lifting $\mathcal{C}$ which is a vector bundle morphism always exists and the path lifting property is satisfied.

2.3. C. Ehresmann has given an equivalent definition of a principal connection by means of a "connection form", i.e. a 1-form on $P$ with values in the Lie algebra $\mathcal{G}$ of $G$.

First he considers the 1 -form $\varpi$ on $V P=\operatorname{ker} T \pi$, this form being independent of the choice of the connection. For any $z \in P$, the form $\varpi$ associates to the vector $z+d z$ element of $V P$, the vector

$$
\varpi(z+d z)=z^{-1}(z+d z)
$$

The vector $\varpi(z+d z)$ being tangent to $G$ at $e$, can be identified with an element of $\mathcal{G}$.

The form $\varpi$ satisfies the relation

$$
\varpi((z+d z) s)=s^{-1}\left(z^{-1}(z+d z)\right) s \quad \text { for any } \quad s \in G,
$$

which can be written

$$
R_{s}^{*} \varpi=\operatorname{Ad}_{s^{-1}} \circ \varpi,
$$

$R_{s}$ meaning the right translation by $s$ on $P$. 
The vertical vectors $z+d z$ and $z^{\prime}+d z^{\prime}$ are said to be equipollent if they have the same image by $\varpi$. Hence we get a trivialization $V P \rightarrow P \times \mathcal{G}$ defined by $z+d z \mapsto(z, \varpi(z+d z))$. The invariant vertical vector fields (whose image by $\varpi$ is constant) are the fundamental vector fields associated to the action of $G$.

Consider now a principal connection on $(P, \pi, M)$; the horizontal distribution $\mathcal{H}$ defines a splitting of $T P$ into the direct sum $T P=V P \oplus \mathcal{H}$; hence C. Ehresmann defines a 1-form $\omega$ on $P$ by $\omega(z+d z)=\varpi(z+\overline{d z})$ where $\overline{d z}$ is the projection of $d z$ on $V P$.

The form $\omega: T P \rightarrow \mathcal{G}$ satisfies the conditions

a) the restriction of $\omega$ to $V P$ is the form $\varpi$;

b) $\omega((z+d z) s)=s^{-1} \omega(z+d z) s$.

The form $\omega$ is the connection form of the given principal connection.

Conversely if a 1-form $\omega: T P \rightarrow \mathcal{G}$ satisfies the conditions a) and b), its kernel ker $\omega$ is a distribution defining a principal connection.

2.4. C. Ehresmann has given a definition of the Cartan "generalized spaces" in terms of fiber bundles [17].

A "generalized space" is a fiber bundle $E(M, F, \widehat{G}, \widehat{P})$ associated to a principal $\widehat{G}$ bundle $\widehat{P}$, satisfying the conditions

a) the fiber $F$ is a homogeneous space $\widehat{G} / G$ (where $G$ is a closed subgroup of $\widehat{G}$ ); $\operatorname{dim} F=$ $\operatorname{dim} M$;

b) there exists a section $\sigma: M \rightarrow E$.

These conditions imply the existence of a principal $G$-bundle $P$, subbundle of $\widehat{P}$. The bundle $P$ is the set of all diffeomorphisms from $\widehat{G} / G$ to the fibers of $E$ which send the fixed point $a_{0}$ of $\widehat{G} / G$ onto a point of the section $\sigma(M)$.

Then C. Ehresmann introduces the notion of a Cartan connection. Such a Cartan connection is defined by a 1 -form $\omega$ on $P$, with values in the Lie algebra $\widehat{\mathcal{G}}$ of $\widehat{G}$ such that:

a) the restriction of $\omega$ to the vertical bundle $V P$ is the form $\varpi$ defined in 2.3;

b) $\omega((z+d z) s)=s^{-1}\left(z^{-1}(z+d z)\right) s$ for any $s \in G$;

c) for any $z \in P$, the relation $\omega(z+d z)=0$ implies $d z=0$.

Hence $\omega$ defines a parallelism on $P$.

C. Ehresmann has proved that any "generalized space" admits Cartan connections. Moreover each Cartan connection induces a soldering of $E$ onto the base $M$, i.e. there exists an isomorphism from the vector bundle of all tangent vectors to the fibers with origin at a point of $\sigma(M)$ onto the tangent bundle $T M$. Conversely C. Ehresmann proves that a soldering on a "generalized space" induces a Cartan connection. For more details see [37].

A Cartan connection form on $P$ is not a principal connection on $P$ because $\omega$ takes its values in $\widehat{\mathcal{G}}$ and not necessarily in $\mathcal{G}$. But any Cartan connection on $P$ can be extended to a principal connection $\widehat{\omega}$ on the $\widehat{G}$-bundle $\widehat{P}$. Indeed $\widehat{P}$ can be identified with $P \times{ }_{G} \widehat{G}$, quotient of $P \times \widehat{G}$ by the equivalence relation $(z, s) \sim\left(z g, g^{-1} s\right)$ for $z \in P, s \in \widehat{G}, g \in G$. 
The form $\omega-\eta$ on $P \times \widehat{G}$ (where $\eta$ is the Maurer-Cartan form on $\widehat{G}$ ) is the pull back (by the morphism $\Lambda: P \times_{G} \widehat{G} \rightarrow \widehat{P}$ ) of a connection form $\widehat{\omega}$ on $\widehat{P}$. See [35].

The Cartan connection on $P$ is said to be integrable if the connection $\widehat{\omega}$ is integrable. C. Ehresmann has shown that in this case, there exists a local development of $M$ onto the fiber $F=\widehat{G} / G$; so $M$ is locally homogeneous; C. Ehresmann has proved that, when $M$ is compact (or at least complete) and simply connected then $M$ is homogeneous. The manifold is said to be complete if any smooth path on $F$ admits a development onto $M$.

In his paper [37], C. Marle has explained the notion of development. He also has studied the affine, projective and conformal connections, with applications to Mechanics and Physics.

The notations used by C. Ehresmann were useful but they have to be employed carefully. For instance if we consider a principal $G$-bundle $P$, the expression $\left(z^{\prime}+d z^{\prime}\right) z^{-1}$ is meaningful for $z \in P, z^{\prime}+d z^{\prime} \in T P$. The form $\gamma$ such that $\gamma(z+d z)=(z+d z) z^{-1}$ takes its values in the Lie algebroid of the groupoid $P P^{-1}$.

The expression $z^{-1}\left(z^{\prime}+d z^{\prime}\right)$ is meaningful only if $z^{\prime}+d z^{\prime}$ is vertical and if $z=z^{\prime} s$ for some $s \in G$.

The form $\varpi$ such that $\varpi(z+d z)=z^{-1}(z+d z)$ takes its values in the Lie algebra of $G$.

The notations are not convenient for higher order calculus on manifolds. It is why C. Ehresmann introduced the notions of jet soon after he wrote his paper on connections.

\section{Semi-holonomic jets [21]}

3.1. Utilizing the terminology of Mechanics, C. Ehresmann has defined the non-holonomic jets by iteration of 1-jets. The semi-holonomic jets are non-holonomic jets satisfying some extra conditions. They are obtained from usual jets (called holonomic jets) in "forgetting" the Schwarz symmetry lemma for jets of order $\geq 2$. The semi-holonomic jets are found in many situations in Differential Geometry.

Let $\pi: E \rightarrow M$ be a surjective submersion (also called fibered manifold) and $J_{1} E$ be the set of 1-jets of all sections of $\pi$. The second order non-holonomic prolongation is the set $\widetilde{J}_{2} E=J_{1} J_{1} E$. By iteration we define $\widetilde{J}_{q}=J_{1} \widetilde{J}_{q-1} E$. We can also define $\widetilde{J}_{q}(M, N)$ setting $E=M \times N$ and $\pi=\operatorname{pr}_{1}$.

The semi-holonomic prolongation $\bar{J}_{2} E \subset J_{1} J_{1} E$ is defined as follows: a local section $U \subset M \rightarrow J_{1} E$ is said to be adapted at $x \in U$ if $s(x)=j_{x}^{1}(\beta \circ s)$, where $\beta$ is the target map; then the jet $j_{x}^{1} s$ is called semi-holonomic.

As remarked by J. Pradines, the subset $\bar{J}_{2} E \subset \widetilde{J}_{2} E$ may be defined utilizing the commutative diagram

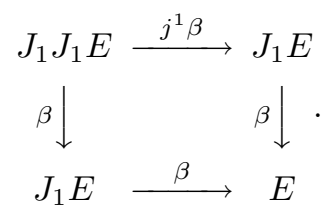

Then

$$
\bar{J}_{2} E=\left\{z_{2} \in J_{1} J_{1} E ; \beta\left(z_{2}\right)=j^{1} \beta\left(z_{2}\right)\right\}
$$


In other terms $\bar{J}_{2} E$ is the inverse image of the diagonal $J_{1} E \times_{M} J_{1} E$ by the map $\left(\beta, j^{1} \beta\right.$ ). If $s=j^{1} f$ (where $f$ is a local section of $E$ ), then $s$ is adapted at each point of its source and $j_{x}^{1} s=j_{x}^{1} j^{1} f=j_{x}^{2} f$. So the holonomic prolongation $J_{2} E$ is contained in $\bar{J}_{2} E$.

By iteration we consider the commutative diagram

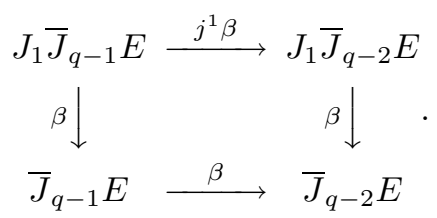

As $\bar{J}_{q-1} E \subset J_{1} \bar{J}_{q-2} E$, the projection $\bar{\pi}_{q-2}^{q-1}: \bar{J}_{q-1} E \rightarrow \bar{J}_{q-2} E$ is the restriction to $\bar{J}_{q-1} E$ of the target map $\beta: J_{1} \bar{J}_{q-2} E \rightarrow \bar{J}_{q-2} E$. The projection $J_{1} \bar{J}_{q-1} E \rightarrow J_{1} \bar{J}_{q-2} E$ is the 1 -jet prolongation of $\beta$. We define

$$
\bar{J}_{q} E=\left\{z_{q} \in J_{1} \bar{J}_{q-1} E ; \beta\left(z_{q}\right)=j^{1} \beta\left(z_{q}\right)\right\} .
$$

The semi-holonomic prolongation $\bar{J}_{q} E$ is a submanifold of $J_{1} \bar{J}_{q-1} E$ and of $\widetilde{J}_{q} E=$ $J_{1} \widetilde{J}_{q-1} E$; the holonomic prolongation $J_{q} E$ is contained in $\bar{J}_{q} E$.

We have also to consider the prolongation $\bar{J}_{q, q-1} E$, inverse image of $J_{q-1} E$ by the projection $\bar{\pi}_{q-1}^{q}=\beta: \bar{J}_{q} E \rightarrow \bar{J}_{q-1} E$ as well as the sesquiholonomic prolongation $\check{J}_{q} E$ defined by

$$
\check{J}_{q} E=\left\{z_{q} \in J_{1} J_{q-1} E ; \beta\left(z_{q}\right)=j^{1} \beta\left(z_{q}\right)\right\} .
$$

This prolongation $\check{J}_{q} E$ is contained in $\bar{J}_{q, q-1} E$.

For $q=2$, then $\bar{J}_{2,1} E$ and $\breve{J}_{2} E$ coincide with $\bar{J}_{2} E$.

Setting $E=M \times N$ and $\pi=\operatorname{pr}_{1}$, we can define $\bar{J}^{q}(M, N)$. In particular all semiholonomic jets from $\mathbb{R}$ to a manifold are holonomic. For instance the elements of TTM whose projections $p$ and $T p$ on $T M$ are equal are the holonomic tangent vectors to $T M$; they define second order differential equations.

\subsection{Examples}

3.2.1. Example 1. Let $(E, \pi, M)$ be a fibered manifold with $\operatorname{dim} M=n$. The prolongation $J_{1} E$ may be considered as the set of all contact $n$-elements in $E$ that are transverse to the vertical bundle $V E$. Then the lifting $C: E \rightarrow J_{1} E$ can be called generalized connection because the path lifting property is not necessarily satisfied. We define the 1-jet extension $j^{1} C: J_{1} E \rightarrow J_{1} J_{1} E$. It is easy to check that the lifting $j^{1} C \circ C$ takes its values in $\bar{J}_{2} E$. Utilizing the Frobenius theorem, we show that the horizontal distribution is completely integrable if and only if $j^{1} C \circ C$ takes its values in the holonomic prolongation $J_{2} E$.

For a holonomic connection of order $q$, i.e. a lifting $C_{q}: J_{q-1} E \rightarrow J_{q} E$, then $j^{1} C_{q} \circ C_{q}$ takes its values in the sesquiholonomic prolongation $\breve{J}_{q+1} E$.

More generally for a regular differntial system $R_{q} \rightarrow M$, where $R_{q} \rightarrow M$ is a fibered submanifold of $J_{q} E \rightarrow M$, we define the sesquiholonomic prolongation of $R_{q}$ by

$$
\check{R}_{q+1}=J_{1} R_{q} \cap \check{J}_{q+1} E .
$$


When $R_{q}$ is diffeomorphic to $R_{q-1}$ (system of finite type), the system $R_{q}$ is integrable if and only if $\check{R}_{q+1}$ coincides with $R_{q}$, according to the Frobenius theorem. By an integrable system we mean a system such that any $z_{q} \in R_{q}$ is the $q$-jet of a solution. A solution is a section $s: U \rightarrow E$ such that $j_{x}^{q} s$ belongs to $R_{q}$ for any $x \in U$.

3.2.2. Example 2. Let $\omega: M \rightarrow T^{*} M$ be a Pfaffian form; then for any $x \in M$, there exists a function $f: U \subset M \rightarrow \mathbb{R}$ such that $f(x)=0, j_{x}^{1} f=\omega(x)$. So the section $\omega$ is adapted at any point $x \in M$ and the 1 -jet $j_{x}^{1} \omega$ belongs to $\bar{T}^{* 2}(M)$ (space of all semi-holonomic 2-jets from $M$ to $\mathbb{R}$ or semi-holonomic 2-covelocities). So we have proved that

$$
J_{1} T^{*} M \text { is diffeomorphic to } \bar{T}^{* 2}(M) .
$$

The jet $j_{x}^{1} \omega$ belongs to $T^{* 2}(M)$ at any $x$ if and only if $\omega=j^{1} f$, i.e. $\omega=d f$ in the neighbourhood of $x$. So $\omega$ must be closed.

In terms of local coordinates, we write in an open subset $U$ of $M$

$$
\omega=\sum_{i} a_{i} d x^{i}
$$

$j_{x}^{1} \omega$ is defined by $a_{1}(x), \ldots, a_{n}(x), \frac{\partial a_{i}}{\partial x^{j}}(x)$.

If we assume $U$ to be simply connected, the 2 -jet is holonomic if and only if

$$
\frac{\partial a_{i}}{\partial x^{j}}=\frac{\partial a_{j}}{\partial x^{i}}
$$

3.2.3. ExAmple 3. Let $(P, \pi, M)$ be a principal $G$-bundle. The projection $J_{1} P \rightarrow M$ is not necessarily a principal bundle map. But we have constructed a principal bundle $\left(\mathcal{T}_{n} P, \pi^{\prime}, M\right)$ (see [30]). The manifold $\mathcal{T}_{n} P$ (with $\left.n=\operatorname{dim} M\right)$ is the submanifold of $T_{n} P$ (set of all 1-jets from $\mathbb{R}^{n}$ to $P$ with source 0 ), inverse image of the frame bundle $H(M)$ by the projection $T_{n} P \rightarrow T_{n} M$. We have proved that $\mathcal{T}_{n} P$ is diffeomorphic to $J_{1} P \times_{M} H(M)$. The manifold $\mathcal{T}_{n} P$ enjoys also the following property: the gauge groupoid $\Phi^{(1)}$ of $\mathcal{T}_{n} P$ is the prolongation (in the sense of Ehresmann) of the gauge groupoid $\Phi$ of $P$, i.e. $\Phi^{(1)}$ is the set of the 1-jets of all invertible $\alpha$-sections of $\Phi$.

If we consider the principal bundle $\left(M, \operatorname{id}_{M}, M\right)$ we identify $\mathcal{T}_{n} M$ with $H(M)$. We define $\bar{H}^{2}(M)$ (second order semi-holonomic frame bundle) as the submanifold of $\mathcal{T}_{n} \mathcal{T}_{n} H$, inverse image of the diagonal of $\mathcal{T}_{n} M \times \mathcal{T}_{n} M$ by the map $\left(\beta, j^{1} \beta\right)$. As $\beta$ and $j^{1} \beta$ are principal bundles morphisms, it follows that $\bar{H}^{2}(M) \rightarrow M$ is a principal subbundle of $\mathcal{T}_{n} \mathcal{T}_{n} M \rightarrow M$

Any $n$-contact element on $H(M)$ which is transverse to the vertical bundle $V H(M)$ is the image of an element of $\mathcal{T}_{n} H$ (considered also as a linear map). It can be deduced the following property: there exists a natural diffeomorphism

$$
\Psi_{1}: J_{1} H(M) \rightarrow \bar{H}^{2}(M)
$$

inducing a principal bundle structure to the projection $J^{1} H(M) \rightarrow M$.

By iteration, it is possible to prove the existence of the diffeomorphism

$$
\Psi_{q}: \bar{J}_{q} H(M) \rightarrow \bar{H}^{q+1}(M),
$$

where $\bar{H}^{q+1}(M)$ is the semi-holonomic frame bundle of order $q+1$. 
A generalized connection $C: H(M) \rightarrow J_{1} H(M)$ such that $C$ is a principal bundle morphism is a connection in the sense of Ehresmann. The connection is said to be symmetric if $C$ takes its values in $H^{2}(M)$.

It is known that this connection $C$ (symmetric or not) induces a linear connection on the tangent bundle $T M$. We can prove this property as follows. Utilizing the Schwarz lemma for manifolds we prove that there exists a diffeomorphism $T \mathcal{T}_{n} M \Leftrightarrow \mathcal{T}_{n} T M$. As $T \mathcal{T}_{n} M$ may be written $T H(M)$ and $\mathcal{T}_{n} T M$ is diffeomorphic to $J_{1} T(M) \times_{M} H(M)$, we conclude that the bundle $J_{1} T(M) \rightarrow M$ is isomorphic to the Atiyah-Molino bundle of $H(M)$, i.e.

$$
J_{1} T(M) \text { is diffeomorphic to } T H(M) / L_{n} \text { with } L_{n}=G L(n, \mathbb{R}) .
$$

So the connection $C$ on $H$ induces a lifting $T(M) \rightarrow J_{1} T(M)$ and conversely.

In the same way we can prove that $J_{q} T(M)$ is diffeomorphic to $T H^{q}(M) / L_{n}^{q}$, where $L_{n}^{q}$ is the group of $q$-jets of local diffeomorphisms with source and target 0 .

These properties have been proved in [30] utilizing local vector fields.

Similarly $\bar{J}_{q} T(M)$ is diffeomorphic to $T \bar{H}^{q} / \bar{L}_{n}^{q}$.

3.2.4. ExAmple 4. Consider now a $G$-structure on $M$, i.e. a principal $G$-subbundle $H_{G}(M)$ of $H(M)$, where $G$ is a closed subgroup of $G L(n, \mathbb{R})$.

For $q \geq 1$, we may define by iteration the semi-holonomic frame bundle $\bar{H}_{G}^{q+1} \rightarrow$ $M$ of order $q+1$. It is a principal bundle, image of $\bar{J}_{q} H_{G}(M)$ by the diffeomorphism $\bar{J}_{q} H(M) \rightarrow \bar{H}^{q+1}(M)$.

Then the holonomic prolongation of order $q+1$ of $H_{G}(M)$ is the intersection

$$
H_{G}^{q+1}(M)=\bar{H}_{G}^{q+1}(M) \cap H^{q+1}(M) .
$$

The restriction to $H_{G}^{q+1}(M)$ of the projection $H^{q+1}(M) \rightarrow H(M)$ is not necessarily a surjective map on $H_{G}(M)$. When this projection is surjective on $H_{G}(M)$, it can be proved in this case that the projection $H_{G}^{q+1}(M) \rightarrow H_{G}(M)$ is a principal bundle morphism; then the $G$-structure is said to be $q$-integrable.

The 1-integrability means that there exists a connection $C: H_{G}(M) \rightarrow J_{1} H_{G}(M)$ such that $C$ takes its values in $H^{2}(M)$, i.e. is symmetric.

This problem has been studied by C. Ehresmann [6] using semi-holonomic jets and by D. Bernard [3] by other methods. They introduced the so-called structure tensor.

Many mathematicians worked on higher order; among them I. Kolár [26] utilizing semi-holonomic jets, D. Lehmann [28] and P. Molino [38] by other methods.

Instead of principal bundles we could consider their gauge groupoids with their Lie algebroids.

We obtain the condition of $q$-integrability of these groupoids considering their semiholonomic and holonomic prolongations. If $H_{G}$ is $q$-integrable, then its gauge groupoid $\Phi$ (which is a subgroupoid of the groupoid $\Theta(M)$, set of 1-jets of all local diffeomorphisms on $M)$ is $q$-integrable. The converse is not always true.

If $\Phi$ is $q$-integrable then its Lie algebroid is also $q$-integrable. Conversely if $\Phi$ is a Lie subgroupoid of $\Theta(M)$, its Lie algebroid $R_{1}$ is a vector subbundle of $J_{1} T(M)$ (according to a previous remark). Then if $\Phi$ is $\alpha$-connected and if the prolongation $R_{2}=J_{2} T(M) \cap \bar{R}_{2}$ 
is a vector subbundle of $J_{2} T(M)$ and $\bar{R}_{2}$ then $\Phi$ is 1-integrable, i.e. the mapping

$$
\Theta^{2}(M) \cap \bar{\Phi}^{2} \rightarrow \Phi
$$

is surjective.

For more details see [32].

3.3. Affine properties of semi-holonomic bundles. Let $(E, M, \pi)$ be a fibered manifold. We shall denote by $\bar{\pi}^{q}$ the projection $\bar{J}^{q} E \rightarrow M$, by $\bar{\pi}_{k}^{q}$ the projection $\bar{J}_{q} E \rightarrow \bar{J}_{k} E$, for $0 \leq k \leq q$ with the convention $J_{0} E=E$; the vertical bundle $V E$ is as usual the kernel of the projection $T \pi: T E \rightarrow T M$.

The main property of semi-holonomic prolongations is the following theorem.

THEOREM 1. The projection $\bar{\pi}_{q-1}^{q}: \bar{J}_{q} E \rightarrow \bar{J}_{q-1} E$ defines an affine bundle structure whose associated vector bundle is $\left(\bar{\pi}_{0}^{q-1}\right)^{*} L_{E}^{q}\left(\pi^{*} T M, V E\right)$, i.e. the pullback to $\bar{J}_{q-1} E$ of the vector bundle, with base $E$, of the q-linear morphisms from $\pi^{*} T M$ to VE.

The theorem is known for $q=1$. We have to show that if the theorem is true for the projection $\bar{\pi}_{q-2}^{q-1}$, it is true for the projection $\bar{\pi}_{q-1}^{q}$. As $\bar{J}_{q} E \subset J_{1} \bar{J}_{q-1} E$, if we consider $z_{q} \in \bar{J}_{q} E$ and $z_{q}^{\prime} \in J_{1} \bar{J}_{q-1} E$ which have the same projection $z_{q-1}$ on $\bar{J}_{q-1} E$, then it makes sense to define $z_{q}-z_{q}^{\prime}$. We show that $z_{q}^{\prime}$ belongs to $\bar{J}_{q} E$ if $z_{q}-z_{q}^{\prime} \in L\left(T_{x} M, L^{q-1}\left(T_{x} M, V_{y} E\right)\right)$, i.e. $z_{q}-z_{q}^{\prime} \in L^{q}\left(T_{x} M, V_{y} E\right)$, where $x$ is the projection of $z_{q-1}$ on $M$ and $y$ the projection of $z_{q-1}$ on $E$. For more details see [34].

As we deal with finite dimensional manifolds, we may write

$$
L^{q}\left(T_{x} M, V_{y} E\right)=V_{y} E \otimes \otimes{ }^{q} T_{x}^{*} M .
$$

In the case of the prolongations $\bar{J}_{q, q-1} E$ and $\check{J}_{q} E$, the inverse image of $z_{q-1} \in J_{q-1} E$ by the projection $\bar{J}_{q, q-1} E \rightarrow J_{q-1} E$ (resp. $\breve{J}_{q} E \rightarrow J_{q-1} E$ ) is an affine space whose associated vector space is $V_{y} E \otimes \otimes^{q} T_{x}^{*} M$ (resp. $\left.V_{y} E \otimes \odot^{q-1} T_{x}^{*} M \otimes T_{x}^{*} M\right)$, where $\odot$ is the multiplication in the symmetric algebra of the tensor algebra of $T_{x}^{*} M$.

It is possible to find local coordinates on $\bar{J}_{q} E$, any $z_{q} \in \bar{J}_{q} E$ being defined in the neighbourhood of $\pi^{q}\left(z_{q}\right)$ by the functions $x^{i}, y^{\alpha}, y_{j_{1} \ldots j_{q}}^{\alpha}$. For $J_{q} E$, we have the condition: for $k=2, \ldots, j_{q}$ then $y_{\sigma\left(j_{1}\right), \ldots, \sigma\left(j_{k}\right)}^{\alpha}=y_{j_{1}, \ldots, j_{k}}^{\alpha}$ for any permutation on $k$.

For $\bar{J}_{q, q-1} E$ we have this condition only for $k=2, \ldots, j_{q-1}$. For $\check{J}_{q} E \subset \bar{J}_{q, q-1} E$, the $y_{j_{1} \ldots j_{q}}^{\alpha}$ are symmetric with respect of the $(q-1)$ first indices.

In this section we fix an element $z_{q-1} \in J_{q-1} E$. Let $y=\pi_{0}^{q-1}\left(z_{q-1}\right)$ and $x=\pi(y)$. We denote by $z_{q}$ and $z_{q}^{\prime}$ elements of $\bar{J}_{q, q-1} E$ which project on $z_{q-1}$.

Let $S_{y}$ be the projection

$$
V_{y} E \otimes\left(\otimes^{q} T_{x}^{*} M\right) \rightarrow V_{y} E \otimes\left(\odot^{q} T_{x}^{*} M\right)
$$

defined by

$$
S_{y}(v \otimes u)=\frac{1}{q !} \sum_{\sigma \in \mathcal{P}_{q}} v \otimes \sigma u \quad \text { for } \quad v \in V_{y} E, \quad u \in \otimes^{q} T_{x}^{*} M,
$$

$\mathcal{P}_{q}$ being the group of permutations of $[1, q]$.

Let $A_{y}$ be the projection

$$
V_{y} E \otimes\left(\otimes^{q} T_{x}^{*} M\right) \rightarrow \operatorname{ker} S_{y}
$$


defined by $A_{y}(v \otimes u)=v \otimes u-S_{y}(v \otimes u)$.

If $z_{q}^{\prime} \in J_{q}(E)$, then $A_{y}\left(z-z_{q}^{\prime}\right)$ is independent of the choice of $z_{q}^{\prime}$ in $J_{q} E$. So we have defined a mapping $z_{q} \mapsto \mathcal{A}_{z_{q-1}}\left(z_{q}\right)=A_{y}\left(z-z_{q}^{\prime}\right)$. Then the mapping $S_{z_{q-1}}: z_{q} \mapsto$ $z_{q}-\mathcal{A}_{z_{q-1}}\left(z_{q}\right)$ takes its values in $J_{q}(E)$.

These considerations lead to the following theorem (whose complete proof is explained in [34]).

THEOREM 2. Given a fibered manifold $(E, M, \pi)$ there exists for the prolongation $\bar{J}_{q, q-1} E$ a natural contraction $S: \bar{J}_{q, q-1} E \rightarrow J_{q} E$ and a natural involution $\mathcal{I}: \bar{J}_{q, q-1} E \rightarrow \bar{J}_{q, q-1} E$ such that $\mathcal{I}\left(\check{J}_{q} E\right)=\check{J}_{q} E$ and $\left.\mathcal{I}\right|_{J_{q} E}$ is the identity map. These maps $S$ and $\mathcal{I}$ are defined by

$$
S\left(z_{q}\right)=z_{q}-\mathcal{A}\left(z_{q}\right), \quad \mathcal{I}\left(z_{q}\right)=z_{q}-2 \mathcal{A}\left(z_{q}\right),
$$

where $\mathcal{A}$ is a mapping from $\bar{J}_{q, q-1} E$ onto the kernel of the projection $V E \otimes\left(\otimes{ }^{q} \pi^{*} T M\right) \rightarrow$ $V E \otimes\left(\odot \pi^{*} T M\right)$; this map $\mathcal{A}$ vanishes on $J_{q} E$.

REMARK 3. For $q=2$, then $\bar{J}_{2,1} E=\check{J}_{2} E=\bar{J}_{2} E$. The mapping $\mathcal{A}$ takes its values in $V E \otimes\left(\bigwedge^{2} T^{*} M\right)$. We recover the involution introduced by J. Pradines.

The operator $\mathcal{A}$ permits to define the curvature of a connection, obstruction to integrability. For a connection $C: E \rightarrow J_{1} E$ (where $\pi: E \rightarrow M$ is a fibered manifold), we have seen that $j^{1} C \circ C$ takes its values in $\bar{J}_{2} E$. There exists a morphism $\mathcal{A}: \bar{J}_{2} E \rightarrow V E \otimes\left(\bigwedge^{2} \pi^{*} T M\right)$ which vanishes on $J_{2} E$. Combining with the lifting $j^{1} C \circ C$ we get a morphism

$$
\rho: E \rightarrow V E \otimes\left(\bigwedge^{2} \pi^{*} T M\right)
$$

which vanishes if and only if $j^{1} C \circ C$ takes its values in $J_{2} E$.

Similarly for a connection of order $q$, i.e. a lifting $C_{q}: J_{q-1} E \rightarrow J_{q} E$, the curvature is the morphism of fibered manifolds

$$
\rho_{q}: J_{q-1} E \rightarrow V E \otimes\left(\odot^{q-1} \pi^{*} T M\right) \otimes\left(\bigwedge^{2} T^{*} M\right)
$$

3.4. Second order foliations. In a lecture devoted to foliations [18], C. Ehresmann defined in 1950 second order elements of contact and second order foliations. His results may be interpreted in terms of semi-holonomic jets, notion he introduced only in 1954.

Let $\mathcal{G}_{p}(M)$ be the set of all $p$-contact elements on a manifold $M$, of dimension $n$. C. Ehresmann considered the $p$-contact elements on $\mathcal{G}_{p}(M)$ which satisfy the condition: the origin of the contact element on $\mathcal{G}_{p}(M)$ and the projection on $\mathcal{G}_{p}(M)$ of this contact element are identical. This can be explained as follows. The manifold $\mathcal{G}_{p}(M)$ is the total space of a fibration with standard fiber $\mathcal{G}_{p}\left(\mathbb{R}^{n}\right)$, the Grassmannian of the linear $p$-subspaces of $\mathbb{R}^{n}$.

The set of $p$-elements of order 2 is obtained by means of the commutative diagram

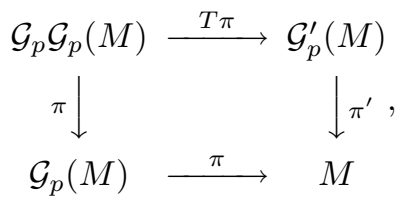


where $\mathcal{G}_{p}^{\prime}(M)$ is the set of contact elements on $M$ of dimension $\leq p$. The set of $p$-contact elements of order 2 on $M$ is, according to C. Ehresmann, the manifold

$$
\overline{\mathcal{G}}_{p}^{2}(M)=\left\{z \in \mathcal{G}_{p} \mathcal{G}_{p}(M) ; \pi(z)=T \pi(z)\right\} .
$$

A second order distribution of p-contact elements is a lifting $c: \mathcal{G}_{p}(M) \rightarrow \overline{\mathcal{G}}_{p}^{2}(M)$. Such a distribution always exists, as the fibration $\overline{\mathcal{G}}_{p}^{2}(M) \rightarrow \mathcal{G}_{p}(M)$ is affine.

The distribution is said to be integrable if for any $z \in \mathcal{G}_{p}(M)$, there exists in the neighbourhood of $\pi(z)$ a $p$-dimensional submanifold of $M$ such that its second order contact elements are the image by $c$ of its tangent spaces. Then the distribution defines a second order foliation. The leaves are the maximal submanifolds which satisfy the above condition.

For instance the totally geodesic submanifolds of a Riemannian manifold with constant curvature are the leaves of a second order foliation. It is the same for the totally geodesic submanifolds of a locally projective space.

3.5. Final remarks. One of the interests of semi-holonomic jets is the possibility of building prolongations of differential systems (considered as fibered submanifolds) which are also fibered manifolds while the holonomic prolongation is not necessarily a manifold.

Among the mathematicians who have investigated semi-holonomic jets are C. Ehresmann pupils (J. Pradines, myself, P. Ver Eecke). A very important contribution has been made by I. Kolář [26] and his coworkers as well as G. Virsik [42]. The theory is linked with the research of natural transformations studied in [27].

J. Pradines [41] has initiated the theory of double vector bundles. He has shown the links between this theory and the theory of non-holonomic and semi-holonomic jets; this theory is also connected with the theory of groupoids and algebroids [36].

\section{References}

[1] C. Albert and P. Dazord, Groupoïdes de Lie et groupoïdes symplectiques, Séminaire Sud Rhodanien à Berkeley (1989), M.S.R.I. Publications 20, Springer-Verlag, BerlinHeidelberg-New York (1991), 1-12. Publications Dep. Math. Lyon (1988), 53-105, (1990), 27-99.

[2] C. Albert and P. Molino, Pseudogroupes de Lie transitifs, Travaux en cours, Hermann, Paris, 1987.

[3] D. Bernard, Sur la géométrie différentielle des G-structures, Ann. Institut Fourier 10 (1960), 151-210.

[4] E. Cartan, Euvres complètes, partie I, Gauthier-Villars, Paris, 1952, partie II, GauthierVillars, Paris, 1953; second edition, CNRS, 1984.

[5] A. Coste, P. Dazord and A. Weinstein, Groupoïdes symplectiques, Publ. Dept. Maht. Lyon 2/A, 1-62, 1987.

[6] C. Ehresmann, Euvres complètes et commentées, Vol. I, parts I and II, Supplement of the Cahiers de Topologie et Géométrie Différentielle, Amiens, 1984.

[7] C. Ehresmann, Sur la topologie de certains espaces homogènes, Ann. of Math. 35 (1934), 396-443 (thèse Paris 1934). 
[8] C. Ehresmann, Sur la notion d'espace complet en géométrie différentielle, CRAS Paris 202 (1936), 2033-2035.

[9] C. Ehresmann, Les groupes de Lie à r paramètres, Sém. de math. Paris IV E.F. (1937), $1-61$.

[10] C. Ehresmann, Sur les espaces analytiques d'un espace de Cartan, CRAS Paris 205 (1938), 1433-1436.

[11] C. Ehresmann and J. Feldbau, Sur les propriétés d'homotopie des espaces fibrés, CRAS Paris 212 (1941), 945-948.

[12] C. Ehresmann, Espaces fibrés associés, CRAS Paris 213 (1941), 762-764.

[13] C. Ehresmann, Espaces fibrés de structures comparables, CRAS Paris 214 (1942), 144-147.

[14] C. Ehresmann, Sur les espaces fibrés associés à une variété différentiable, CRAS Paris 216 (1943), 628-630.

[15] C. Ehresmann and G. Reeb, Sur les champs d'éléments de contact complètement intégrables, CRAS Paris 218 (1944), 955-956.

[16] C. Ehresmann, Sur la théorie des espaces fibrés, Coll. Intern. Topol. Algébrique, CNRS, Paris (1947), 3-15.

[17] C. Ehresmann, Les connexions infinitésimales dans un espace fibré différentiable, Coll. de Topologie, Bruxelles, CBRM (1950), 29-55.

[18] C. Ehresmann, Sur la théorie des variétés feuilletées, Rend. Mat. e Appl. ser. V X-1-2 Rome (1951), 64-83.

[19] C. Ehresmann, Les prolongements d'une variété différentiable, parties I, II, III, IV, V, VI, CRAS Paris 233 (1951), 598-600, 777-779, 1081-1083, 234 (1952), 587-589, 1028-1030, 1424-1425.

[20] C. Ehresmann, Introduction à la théorie des structures infinitésimales et de pseudogroupes de Lie, Coll. Intern. Géom. Diff. Strasbourg CNRS (1953), 97-100.

[21] C. Ehresmann, Jets non holonomes, CRAS Paris 239 (1954), 1762-1764, 240 (1955), 397-399, 1755-1757.

[22] C. Ehresmann, Sur les pseudogroupes de Lie de type fini, CRAS Paris 246 (1958), 360-362.

[23] M.V. Karasev and V.P. Maslov, Nonlinear Poisson Brackets, Transl. Math. Monographs, AMS 1993.

[24] J. Kounheiner, D. Flament, P. Nabonnand and J. J. Szczeciniarz, Géométrie au XX-ème siècle, Hermann, Paris, 2005.

[25] J. Kumpera and D. Spencer, Lie equations, Ann. of Math. Studies 75, Princeton Univ. Press, Princeton, 1972.

[26] I. Kolář, Natural operations with connections on second order frame bundles, Topics in Diff. Geom. (Debrecen 1984), Coll. Math. Soc. János Bolyai, 46, 715-732.

[27] I. Kolář, P. Michor and J. Slovák, Natural Operations in Differential Geometry, Springer Verlag, 1993.

[28] D. Lehmann, Sur l'intégrabilité des G-structures, Symp. Math. X Conv. Geom. Diff Roma 1971, 127-140.

[29] P. Libermann, Sur le problème d'équivalence des structures infinitésimales régulières, thesis Strasbourg 1953, Ann. Math. Pura Appl. 36 (1954), 27-120.

[30] P. Libermann, Prolongements des fibrés principaux et groupoïdes différentiables, Sémin. Analyse globale Montréal (1969), 59-93.

[31] P. Libermann, Parallélismes, Journ. Diff. Geometry 8 (1973), 511-539.

[32] P. Libermann, Remarques sur les systèmes différentiels, Geom. and Diff. Geom. Haifa (1979), Lecture Notes in Math. 792 (1979), and 3rd Colloque sur les catégories, Cahiers Top. and Geom. Diff. XXIII-1 (1982), 55-72. 
[33] P. Libermann, Lie algebroids and Mechanics, Arch. Math. 32 (1996), 147-162.

[34] P. Libermann, Introduction to the theory of semi-holonomic jets, Arch. Math. 33 (1997), 173-189.

[35] P. Libermann, Cartan connections and momentum maps, in: Banach Center Publ. 59, Warszawa, 2003, 211-221.

[36] K. C. H. Mackenzie, General Theory of Lie Groupoids and Lie Algebroids, Cambridge University Press, Cambridge, 2005.

[37] C.-M. Marle, The works of Charles Ehresmann on connections: from Cartan connections to connections on fibre bundles, this volume.

[38] P. Molino, Théorie des G-structures, problème d'équivalence, Lecture Notes in Math., Springer, Berlin, 1978.

[39] J. Pradines, Théorie de Lie pour les groupoïdes différentiables, CRAS Paris I 263 (1966) 907-910, II 264 (1967) 254-258.

[40] J. Pradines, Troisième théorème de Lie pour les groupoïdes différentiables, CRAS Paris 267 (1968).

[41] J. Pradines, Fibrés vectoriels doubles et calcul des jets non holonomes, Esquisses math. 29, Amiens, 1977.

[42] G. Virsik, On the holonomy of higher order connections, Cahiers Top. Géom. Diff. 12 (1971), 197-212. 